This article was published as: Physical Review E 96 (2), 022605, 2017

DOI: 10.1103/PhysRevE.96.022605 


\title{
Shear elasticity of isotropic magnetic gels
}

\author{
M.T.Lopez-Lopez ${ }^{\mathrm{a}, \mathrm{b}}$, D.Yu.Borin ${ }^{\mathrm{c}}$, A.Yu.Zubarev ${ }^{\mathrm{d}, \mathrm{e}^{*}}$, \\ ${ }^{a}$ Departamento de Física Aplicada, Facultad de Ciencias, Universidad de Granada, Campus de \\ Fuentenueva, 18071 Granada, Spain. \\ ${ }^{b}$ Instituto de Investigatacion Biosantaria ibs. GRANADA, Spain \\ ${ }^{c}$ Chair of Magnetofluiddynamics, Measuring and Automation Technology, TU Dresden, 01069, Germany \\ ${ }^{d}$ Ural Federal University, Lenina Ave 51, 620083, Ekaterinburg, Russia \\ ${ }^{e}$ M.N. Mikheev Institute of Metal Physics of the Ural Branch of the Russian Academy of Sciences, S.Kovalevskoy, \\ 18, 620990, Ekaterinburg, Russia
}

\begin{abstract}
The paper deals with theoretical study of effective shear modulus of a magnetic gel, consisting of magnetizable particles randomly and isotropically distributed in an elastic matrix. Effect of external magnetic field on the composite modulus is focus of our consideration. We take into account that magnetic interaction between the particles can induce their spatial rearrangement and lead to internal anisotropy of the system. Our results show that, if this magnetically induced anisotropy is insignificant, the applied field reduces the total shear modulus of the composite. Strong anisotropy can qualitatively change the magnetomechanic effect and induce increase of this modulus with the field.
\end{abstract}

\section{Introduction.}

Composites of fine magnetic particles in soft polymer matrixes (named ferrogels, magnetic elastomers, magnetorheological elastomers) attract considerable interest of researches and engineers because of rich set of unique physical properties, valuable for many industrial and bio-medical applications [1-13].

One of the qualities of these systems, interesting both from scientific and practical points of view, is their ability to change mechanical properties and behavior under the action of external magnetic field. Uniaxial elongation and magnetostriction of magnetic gels have been studied in many works (see, for example [13-20]). The shear effects in the composites with the particles, united in the linear chain-like aggregates, have been studied in [21-23]. The general conclusion of these works is that an external magnetic field can significantly increase the shear modulus of these composites.

The chain-like aggregates are created on the stage preceding the composite curing, by application of an external magnetic field (field of polymerization) to the suspension of the magnetic particles in the liquid polymer. At the same time, very often magnetic gels are 
synthesized without the field. In this case the spatial disposition of the particles is rather random and isotropic (see, for example, $[15,17,24]$ ).

Experiments $[15,17]$ demonstrate that, in soft gels, magnetic field can induce the spatial rearrangement of the particles and even lead to their unification into heterogeneous structures, aligned along the field. Obviously, these internal transformations can lead to significant change of the macroscopic properties of the composite materials.

The aim of this work is theoretical study of effect of external magnetic field on the shear elastic modulus of magnetic gels with initially homogeneous and isotropic distribution of magnetizable spherical particles in a continuous matrix. The field induced rearrangement of the particles and anisotropy of their dispositions is in focus of our consideration.

The matrix is supposed elastic with the linear law of deformation and incompressible. It should be noted that the approximation of incompressibility is fulfilled not for all gels. However it allows us to restrict calculations and to get the final results in the quite transparent forms. Analysis of effects of the composite compressibility can be considered as a natural generalization of this model.

The principal problem of the theory of composite materials is analysis of cooperative effects of the multiparticle interactions. Usually these effects are taken into account by using various empirical and semi empirical methods, whose accuracy, and even qualitative adequacy, a' priory, is unknown [25].

In order to achieve clear understanding of the microscopic nature of the physical properties of magnetic gels as well as their macroscopic behavior, mathematically rigorous models, free from intuitive and heuristic constructions, must be developed. In this work we consider a gel with low or moderate concentration of spherical particles of the magnetic filler. This allows us to use the regular approximation of the pair interaction between the particles and to avoid semi empirical intuitive hypothesis with uncontrolled accuracy. We believe that this approach can be considered as necessary robust background for development of the models for more concentrated systems where the multiparticle effects are very significant.

The paper is organized as follows: in the section II we discuss the main points of the physical model; in sections III and IV we consider the particles relative displacements, as consequences of the macroscopic shear of the composite and the action of the applied field. In the section $\mathrm{V}$ the estimates of the composite shear modulus are presented. 


\section{Physical model and main approximations.}

We consider a system of identical spherical non Brownian magnetizable particles embedded in an elastic continuous medium. For maximal simplification of the mathematics, we restrict ourselves by the case of the moderate field and suppose that the linear law of the particles magnetization if fulfilled.

Let the composite be placed in a uniform magnetic field $\mathbf{H}$ and experiences small shear deformation in the plane, perpendicular to the field (see Fig.1).

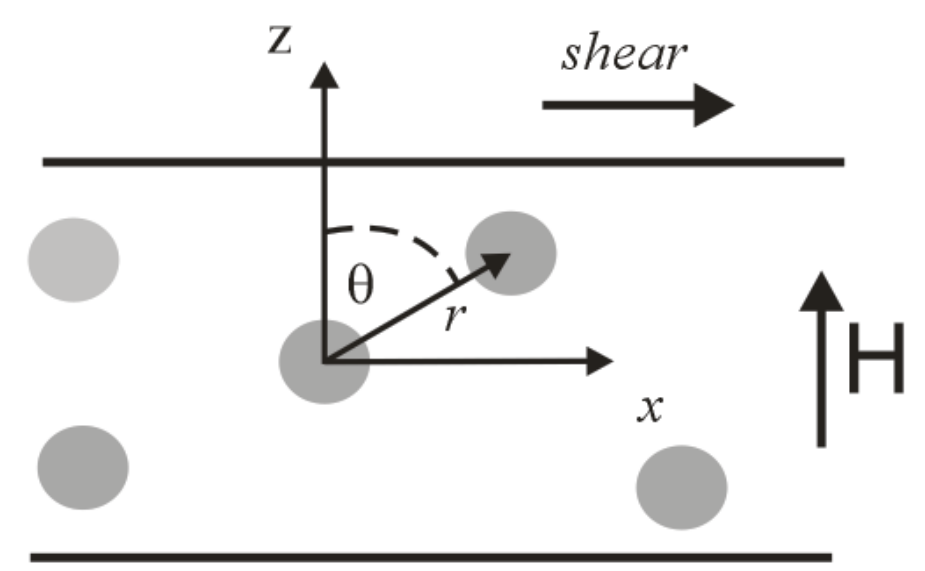

Fig.1. Illustration of the system under consideration. The gradient of the shear displacement is directed along the axis $O z$. The coordinate axis $O y$ is not shown for simplicity.

It is convenient to introduce the Cartesian coordinate system with the axis $O z$ in the field direction and the axis $O x$ in the direction of the shear. By using the well known results of the theory of magnetizable media (see, for example, [26,27]), as well as the mathematical similarity between the stationary Navier - Stokes equation at low Reynolds and the Lame equation of deformation of an elastic poorly compressible medium [25,], one can present the needed component of macroscopic (measurable) stress $\boldsymbol{\sigma}$ in the composite as:

$\sigma=\sigma_{e l}+\sigma_{m}$

$\sigma_{e l}=G_{e l} \gamma ; \quad \sigma_{m}=\frac{1}{2} \varphi \mu_{0}<M_{x}>H$

$\sigma=\sigma_{x z}$

Here $\sigma_{e l}$ is the shear stress in the elastic composite with the hard particles; $G_{e l}$ is the corresponding shear modulus of the composite; $\sigma_{m}$ is the part of the total stress, provided by the applied magnetic field. The magnitude $M_{x}$ is the corresponding component of a particle 
magnetization; the angle brackets mean averaging over all relative positions of the particles in the composite; $\quad \gamma=\frac{\partial u_{x}}{\partial z} ; u_{x}$ is the component of the macroscopic (measurable) vector $\boldsymbol{u}$ of the composite displacement; $\varphi$ is volume concentration of the particles, $\mu_{0}$ is the vacuum magnetic permeability.

By using the Batchelor and Green results [28] of effective viscosity of moderately concentrated suspension, as well as the identity between the Navier-Stokes and Lame equations, one can estimate the modulus $G_{e l}$ as:

$G_{e l}=G_{0}\left(1+2.5 \varphi+5.2 \varphi^{2}\right)$

Here $G_{0}$ is the shear modulus of the pure host matrix. Usually this formula leads to good agreement with experiments till the concentrations $\varphi \sim 10-15 \%$.

One needs to note that, in principle, the solid particles can change the conformation of the macromolecules of the host polymer and, therefore, can change elastic modulus the matrix. The possibility of these transformations, induced by the chemical interaction of the ions, dissolved from the particles surface, and the polymer macromolecules, as well as effect of this interaction on the mechanic properties of the composites, has been discussed, for example, in [29]. Here we will neglect these effects and focus on the mechanic and magnetic interactions between the particles.

Our main goal now is to determine the component $\left\langle M_{x}\right\rangle$ of the particle magnetization. In order to get the mathematically rigorous results, we will estimate $\left\langle M_{x}\right\rangle$ taking into account interaction only between two particles, ignoring the effect of any third one. It should be noted that the Batchelor - Green formula (2) has been derived in the framework of the pair approximation, taking into account mechanic interaction between the particles through the perturbations of the carrier medium.

Let us consider two particles and put the origin of the Cartesian coordinate system, shown in Fig.1, in the center of one of them. We will denote the radius vector of the center of the second particle by $\boldsymbol{r}$.

The component $M_{x}$ of magnetization of each particle, shown in Fig.1, appears because of mutual magnetization of the particles. The simplest way to take this interaction into account is to use the well known dipole-dipole approximation. However, this approach describes quite well magnetic interaction between the particles only when they are far from each other and the distance $r$ between their centers significantly exceeds diameter $d$ of the particle. At the same time, the 
effects of the mutual magnetization are especially strong at the close dispositions of the particles, where their magnetic interaction is multipolar. We will estimate the energy $U(\boldsymbol{r})$ of the particles interaction by using the extrapolation formula obtained in [30] from the results of numerical study of the problem on two linearly magnetizable particles:

$$
U=-3 \mu_{0} \mu_{f} H^{2} v \sum_{k=3}^{7}\left(\frac{\alpha-1}{\alpha+2}\right)^{p_{k}}\left[\frac{a_{k}}{\left(\rho-b_{k}\right)^{k}}+\frac{c_{k}}{\left(q-d_{k}\right)^{k}} \cos ^{2} \theta\right]
$$

Here $\rho=2 r / d ; \alpha=\mu_{p} / \mu_{f}, \mu_{p}$ and $\mu_{f}$ are the relative magnetic permeability of the particle and the host matrix respectively; $v=\pi d^{3} / 6$ is the particle volume; $p_{k}, a_{k} \ldots d_{k}$ are parameters, whose values are tabulated in [30]. For $\rho>>1$ the fit formula (4) coincides with the well known relation for the energy of dipole-dipole approximation.

The $y$-component of the torque $\boldsymbol{\Gamma}$, acting on the cluster of the particles, can be calculated from two general relations. On the one hand $\Gamma_{\mathrm{y}}=-\frac{\partial U}{\partial \theta} \cos \phi[30,31]$ ( $\phi$ is the azimuthal angle, not shown in Fig.1 for brevity); on the other one $\Gamma_{\mathrm{y}}=-2 \mu_{0} v M_{x} H$ [27] (multiplier 2 appears here because we deal with a cluster, consisting of two particles). Equating these relations, taking into account the relations $\mu_{f}=1, \alpha \gg 1$, fulfilled for typical magnetic gels and elastomers, we find $M_{x}$ for the given relative disposition of the particles:

$M_{x}=3 H \sum_{k=3}^{7} \frac{c_{k}}{\left(\rho-d_{k}\right)^{k}} \sin \theta \cos \theta \cos \phi$

Let $g(\boldsymbol{r})$ be the pair distribution function over relative positions of the particles. For the convenience we suppose that the following normalization condition

$g \rightarrow 1$ when $r \rightarrow \infty$

is held.

In the frame of the pair approximation, the average magnetization of a particle can be presented as:

$<M_{x}>=n \int M_{x}(\boldsymbol{r}) g(\boldsymbol{r}) d \boldsymbol{r}, n=\frac{\varphi}{v}$

Here $n$ is number of the particles in a unit volume of the composite, $v$ again, is volume of the particle.

The distribution function $g$ can be presented as: $g=g_{0}+\delta g$, where $g_{0}$ corresponds to the isotropic the non deformed composite before the field application; $\delta g$ reflects change of the 
function because of the particles rearrangement and the sample deformation. In the isotropic composite, where $g_{0}$ depends only on the absolute value $r$ of the radius vector $\boldsymbol{r}$, the equality

$\int M_{x}(\boldsymbol{r}) g_{0}(\boldsymbol{r}) d \boldsymbol{r}=0$

is held. Therefore:

$<M_{x}>=\frac{\varphi}{v} \int M_{x}(\boldsymbol{r}) \delta g(\boldsymbol{r}) d \boldsymbol{r}$

The function $\delta g$ can be determined from the following equation [31]:

$\delta g=-\operatorname{div}(g \boldsymbol{w})$

Here $\boldsymbol{w}$ is vector of the relative displacement of the particles, which is determined by two factors - by the particles rearrangement under magnetic interaction between them and by the macroscopic shear of the composite as well. We will consider separately these displacements and the corresponding changes $\delta g$ of the distribution function $g$.

\section{Magnetically induced displacement of the particles.}

Let us suppose that the sample does not undergo the macroscopic deformation and the particles rearrangement takes place because of their magnetic interaction. By using (4), we determine the components of the force $\boldsymbol{F}=-\nabla U$ of magnetic interaction between two particles. The vector of their relative displacement $\boldsymbol{w}_{m}$, induced by this interaction, can be determined from the theory [33] of mutual motion of two particles in a suspension combined with the mathematical identity of the Navier-Stokes and Lame equations:

$\boldsymbol{w}_{m}=\widehat{\boldsymbol{\beta}} \cdot \boldsymbol{F}$

Here $\widehat{\boldsymbol{\beta}}$ is tensor of the particles displacement. According to [33], the Cartesian components of this tensor can be presented as:

$$
\begin{aligned}
& \beta_{i i}=\beta_{0}\left[\left(G_{B}-H_{B}\right) \frac{x_{i}^{2}}{r^{2}}+H_{B}\right] \\
& \beta_{i j}=\beta_{0}\left(G_{B}-H_{B}\right) \frac{x_{i} x_{j}}{r^{2}} \\
& \beta_{0}=\frac{2}{3 \pi G_{0} d}
\end{aligned}
$$


Here $G_{0}$ again is the shear modulus of the host elastic matrix; $i, j=x, y, z ; G_{B}(r)$ and $H_{B}(r)$ are some functions of the distance $r$ between centers of the particles. Explicit forms of these functions, valid for all values of $r$, are unknown. Their asymptotic approximations are given in [33]:

$G_{B} \approx 2(\rho-2) ; \quad H_{B} \approx 0.401$, when $\rho \rightarrow 2$

$G_{B} \approx 1-\frac{3}{2} \rho^{-1}+\rho^{-3}-\frac{15}{4} \rho^{-4}+O\left(\rho^{-6}\right) ; H_{B} \approx 1-\frac{3}{4} \rho^{-1}-\frac{1}{2} \rho^{-3}+O\left(\rho^{-6}\right)$,

when $\rho \rightarrow \infty$

The dimensionless distance $\rho$ between the particles is defined in (4). Numerical dates for $G_{B}(\rho)$ and $H_{B}(\rho)$ are tabulated in [33].

In the spherical coordinate system, with the radius $r$, polar and azimuth angles $\theta$ and $\phi$ respectively ( $x=r \sin \theta \cos \phi ; \quad y=r \sin \theta \sin \phi ; \quad z=r \cos \theta)$, after simple, but cumbersome calculations, we get the following relations for the components of the displacement vector $\boldsymbol{w}_{m}$ :

$w_{m r}=\kappa \beta_{0} \mu^{\prime}(r) G_{B}(r)\left(3 \cos ^{2} \theta-1\right)$,

$w_{m \theta}=-6 \kappa \beta_{0} \frac{\mu(r)}{r} H_{B}(r) \sin \theta \cos \theta$,

$w_{m \phi}=0$

Here

$\mu(r)=\sum_{k=3}^{7} \frac{c_{k}}{\left(\rho-d_{k}\right)^{k}}, \mu^{\prime}(r)=\frac{d \mu}{d r}, \kappa=3 \mu_{0} H^{2} v$

By using (7), in the first approximation with respect to the displacement $\boldsymbol{w}_{m}$, the change $\delta g_{m}$ of the distribution function can be determined as:

$\delta g_{m}=-\operatorname{div}\left(g_{0} \boldsymbol{w}_{\boldsymbol{m}}\right)$

Combining (11) and (12), one can obtain after calculations:

$\left.\delta g_{m}=-\kappa \beta_{0} f(r)\left(3 \cos ^{2} \theta\right)-1\right)$

$f(r)=\frac{1}{r^{2}} \frac{d}{d r}\left(r^{2} g_{0} G_{B} \mu^{\prime}\right)-6 g_{0} \frac{\mu}{r^{2}} H_{B}$

The function $\delta g_{m}$ describes the structural anisotropy which appears in the non deformed composite under the action of the applied field. 


\section{The particles displacement due to the composite shear.}

Let us suppose now that the sample experiences macroscopic shear in the direction $O x$ and gradient of the sample displacement is directed along $O z$. The vector $\boldsymbol{w}_{\gamma}$ of the corresponding relative displacement of the particles again can be determined by using the identity of the Navier - Stokes and Lame equations [25], as well as the relation [28] for the relative motion of two particles in a suspension. In the spherical coordinate system the result reads:

$w_{\gamma r}=\gamma r\left(1-A_{B}\right) \sin \theta \cos \theta \cos \phi$

$w_{\gamma \theta}=\gamma r\left(\cos ^{2} \theta+\frac{1}{2} B_{B}\left(\sin ^{2} \theta-\cos ^{2} \theta\right)\right) \cos \phi$

$w_{\gamma \phi}=-\gamma r\left(1-\frac{1}{2} B_{B}\right) \cos \theta \sin \phi$

Here $\gamma$ is the macroscopic dimensionless shear of the sample, $A_{B}(r)$ and $B_{B}(r)$ are functions of $r$. Their explicit forms for the whole range of $r$ have not been determined in literature; asymptotic relations are given in [28] as:

$A_{B} \approx 1-4.077(\rho-2) ; \quad B_{B} \approx 0.406$, when $\rho \rightarrow 2$

$A_{B} \approx 5 \rho^{-3}-\frac{40}{3} \rho^{-5}+25 \rho^{-6} ; B_{B} \approx \frac{16}{3} \rho^{-5}$, when $\rho \rightarrow \infty$

In the range $2<\rho<20$ the numerical values of $A_{B}$ and $B_{B}$ are tabulated in [28].

Note that the equality $A_{B}(r)=1$ is held at $r=d$ (i.e. at $\rho=2$ ). Thus the condition $w_{\gamma r}(r=$ $d)=0$ of the particles non intersection is fulfilled in (14). At the same time the components $w_{\gamma \theta}$ and $w_{\gamma \phi}$ are not zero at $=d$ (i. e. $\rho=2$ ). This means that the particles can slip over each other being in the physical contact.

By using eq.(7), in the first approximation with respect to $\gamma$, one can present the change $\delta g_{\gamma}$ of the distribution function, produced by the macroscopic shear, as:

$\delta g_{\gamma}=-\operatorname{div}\left(\left(g_{0}+\delta g_{m}\right) \boldsymbol{w}_{\gamma}\right)$

Combining $(13,14)$ and $(16)$, after some transformations we get:

$\delta g_{\gamma}=\delta g_{\gamma}^{(1)}+\delta g_{\gamma}^{(2)}$

$\delta g_{\gamma}^{(1)}=-\operatorname{div}\left(g_{0} \boldsymbol{w}_{\gamma}\right)=-\gamma\left[\frac{1}{r^{2}} \frac{d}{d r}\left(r^{3}\left(1-A_{B}\right) g_{0}\right)-3 g_{0}\left(1-B_{B}\right)\right] \sin \theta \cos \theta \cos \phi$ 


$$
\begin{aligned}
& \delta g_{\gamma}^{(2)}=-\operatorname{div}\left(g_{m} \boldsymbol{w}_{\gamma}\right)= \\
& =\kappa \beta_{0} \gamma\left[\frac{1}{r^{2}} \frac{d}{d r}\left(r^{3}\left(1-A_{B}\right) f\right)\left(3 \cos ^{2} \theta-1\right)\right. \\
& \left.\quad+3 f\left(1-5 \cos ^{2} \theta-B_{B}\left(2-5 \cos ^{2} \theta\right)\right)\right] \sin \theta \cos \theta \cos \phi
\end{aligned}
$$

The function $f(r)$ is defined in eq. (13). The term $\delta g_{\gamma}^{(1)}$ corresponds to mutual rearrangement of the particles due to the macroscopic shear of the composite; the term $\delta g_{\gamma}^{(2)}$ - due to the combination of the shear and magnetic interaction between the particles.

Substituting $\delta g=\delta g_{m}+\delta g_{\gamma}$ into (6), taking into account (5) and the equality

$\frac{\varphi}{v} \int M_{x}(\boldsymbol{r}) \delta g_{m}(\boldsymbol{r}) d \boldsymbol{r}=0$,

we come to the relation:

$<M_{x}>=<M_{x}>^{(1)}+<M_{x}>^{(2)}$

$<M_{x}>^{(1)}=\frac{\varphi}{v} \int M_{x}(\boldsymbol{r}) \delta g_{\gamma}^{(1)}(\boldsymbol{r}) d \boldsymbol{r}, \quad<M_{x}>^{(2)}=\frac{\varphi}{v} \int M_{x}(\boldsymbol{r}) \delta g_{\gamma}^{(2)}(\boldsymbol{r}) d \boldsymbol{r}$

By using (17) in (18), one can get after some transformations:

$<M_{x}>^{(1)}=-\frac{3}{5} \gamma H \varphi J$

$J=\int_{0}^{\infty} \mu(\rho)\left[\frac{d}{d \rho}\left(g_{0} \rho^{3}\left(1-A_{B}(\rho)\right)\right)-3 \rho^{2}\left(1-B_{B}(\rho)\right)\right] d \rho$

and

$<M_{x}>^{(2)}=-\frac{3}{35} \gamma H \varphi \frac{\kappa \beta_{0}}{a^{2}} \int_{0}^{\infty} f(\rho) q(\rho) \rho^{2} d \rho$

$q(\rho)=2 \mu^{\prime}\left(1-A_{B}\right) \rho+3 \mu\left(8-B_{B}\right)$

The function $f(\rho)$ presents the function $(r)$, defined in (14), after replacement $r$ to $\rho$. Prime here and below means derivation over $\rho$.

By using the explicit form (14) for the function $f$, integrating by part in (19) and (20), taking into account that

$g_{0}=0$ when $\rho<2$ 
$\mu \rightarrow \frac{3}{\rho^{3}}, g_{0} \rightarrow 1, A_{B} \rightarrow 0, \quad f(\rho) \rightarrow 0$ when $\rho \rightarrow \infty$

we come to the following relations:

$J=3-\int_{2}^{\infty} g_{0}\left[\rho^{3}\left(1-A_{B}\right) \mu^{\prime}+3 \rho^{2}\left(1-B_{B}(\rho)\right) \mu\right] d \rho$

and

$<M_{x}>^{(2)}=\frac{4}{35} \mu_{0} \gamma \frac{H^{3}}{G_{0}} \varphi K$

$K=\int_{2}^{\infty} g_{0}\left(r^{2} \mu^{\prime} q^{\prime} G_{B}-6 \mu H_{B} q\right) d \rho$

\section{Results and discussion.}

In order to calculate the integrals in $(21,22)$, one needs to determine the initial distribution function $g_{0}$ as well as the functions $A_{B}(\rho), B_{B}(\rho), G_{B}(\rho)$ and $H_{B}(\rho)$.

We will chose the distribution function $g_{0}$ of the hard spheres in the isotropic non deformed composite by using the simplest form, which takes into account the steric interaction between the particles and the short range order, created by this interaction [34] :

$$
\begin{gathered}
0, r<d \\
g_{0}=1+8 \varphi\left(1-\frac{3 r}{4 d}+\frac{r^{3}}{16 d^{3}}\right), d<r<2 d \\
1, r>2 d
\end{gathered}
$$

It has been noted that the explicit analytical forms of $A_{B}(\rho), B_{B}(\rho), G_{B}(\rho)$ and $H_{B}(\rho)$ in the whole range of $\rho$ are unknown. Some numerical values of these functions are tabulated in $[28,33]$. However they are given for different magnitudes of $\rho$, that is why these tables are inconvenient for numerical integration in $(21,22)$.

In order to get acceptable estimates for $J$ and $K$, we suggest the following extrapolation forms for the functions $A_{B}(\rho), B_{B}(\rho), G_{B}(\rho)$ and $H_{B}(\rho)$, which coincide with the asymptotic values of the functions (10) and (15) in the corresponding limiting cases with respect to $\rho$ :

$$
\begin{aligned}
& A_{B}(\rho)=\begin{array}{l}
1-4.077(\rho-2), \quad \text { if } 2<\rho<2.13 \\
5 \rho^{-3}-\frac{40}{3} \rho^{-5}+25 \rho^{-6}, \quad \text { if } \rho>2.13
\end{array} \\
& B_{B}(\rho)=\frac{0.406\left(\frac{16}{3}\right)(2 \rho)^{-5}}{\left(\frac{16}{3}\right)(2 \rho)^{-5}+0.406\left(2^{-5}-\rho^{-5}\right)}
\end{aligned}
$$


$G_{B}(\rho)=\frac{2(\rho-2)\left|1-\frac{3}{2} \rho^{-1}+\rho^{-3}-\frac{15}{4} \rho^{-4}\right|}{2(\rho-2)+\left|1-\frac{3}{2} \rho^{-1}+\rho^{-3}-\frac{15}{4} \rho^{-4}\right|}$

$H_{B}(\rho)=\frac{0.401(\rho-1)}{0.401(\rho-2)+1}$

Results of calculations of these functions, by using the extrapolations (22) and the tabulated values of [28,33], are shown in Fig.2.
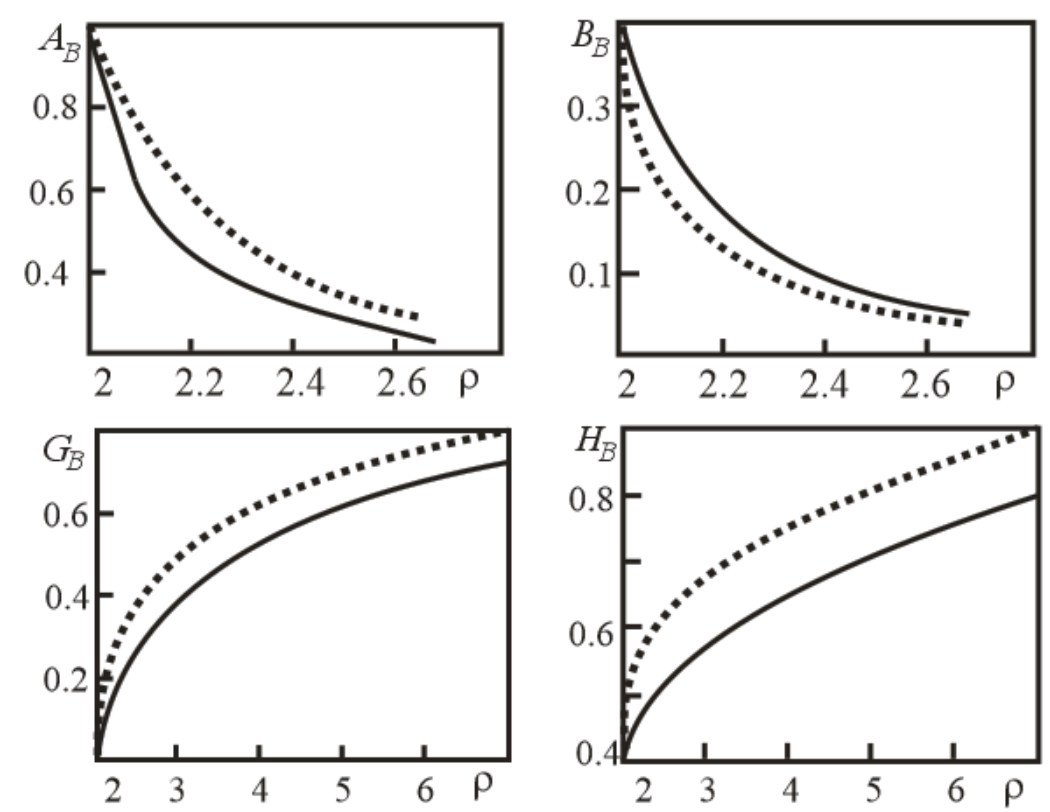

Fig.2. The functions $A_{B}(\rho), B_{B}(\rho), G_{B}(\rho)$ and $H_{B}(\rho)$, calculated by using the forms (24) (solid lines) and the tables of $[28,33]$ (dotted ones).

Comparison of these results allows us to consider the simple extrapolations (22) as quite acceptable approximations for the first estimates of the integrals $J$ and $K$.

Substituting $(23,24)$ into the integrals in $(21,22)$, we get:

$J \approx 2.5-0.213 \varphi$

$K \approx 48+99 \varphi$

One needs to note that parameters $J$ and $K$ are determined by using the form (23) of the distribution function $g_{0}$. The first terms in the right parts of (23) correspond to the step-wise function: 
$g_{0}=\begin{gathered}0, r<2 \\ 1, r>2\end{gathered}$

typical for the ideal gas of the hard spheres. In this approximation $J \approx 2.5 ; K \approx 48$. The second, proportional to the concentration $\varphi$, terms appear in (25) due to the spatial correlations between the spherical particles, mirrored by the term with $\varphi$ in eq. (23).

Combining the relations (1) with $(18,19)$, one can get:

$\sigma_{m}=\sigma_{m}^{(1)}+\sigma_{m}^{(2)}$

$\sigma_{m}^{(1)}=\frac{1}{2} \varphi \mu_{0}<M_{x}>^{(1)} H=G_{m}^{(1)} \gamma$,

$\sigma_{m}^{(2)}=\frac{1}{2} \varphi \mu_{0}<M_{x}>^{(2)} H=G_{m}^{(2)} \gamma$

Here $\sigma_{m}^{(1)}$ and $\sigma_{m}^{(2)}$ are the magnetically induced parts of the total stress $\sigma$, which appear due to the change of the particles mutual disposition, as a consequence, respectively, of the macroscopic shear deformation of the isotropic composite, and because of combination of this deformation with the magnetically induced particles rearrangement. Parameters $G_{m}^{(1)}$ and $G_{m}^{(2)}$ are corresponding parts of the magnetic contributions to the total shear modulus $G$, which can be written as:

$G=G_{e l}+G_{m}^{(1)}+G_{m}^{(2)}$

Taking into account $(19,21,22,25)$ and $(26)$, we come to the following results:

$G_{m}^{(1)}=-0.3 \mu_{0} \varphi^{2} H^{2}(2.5-0.213 \varphi)$

$G_{m}^{(2)}=\frac{2}{35} \varphi^{2} \frac{\mu_{0}^{2} H^{4}}{G_{0}}(48+99 \varphi)$

If the rearrangement of the particles, induced by their magnetic interaction, is insignificant $\left(\left|G_{m}^{(1)}\right|>G_{m}^{(2)}\right.$, i.e. $\left.G_{0} \gg \mu_{0} H^{2}\right)$ the field reduces the total shear modulus $G$ of the composite $\left(G_{m}^{(1)}\right.$ is negative). In the opposite case $\left(\mu_{0} H^{2}>G_{0}\right)$, the magnetically induced anisotropy of the particles dispositions is strong enough $\left(G_{m}^{(2)}>\left|G_{m}^{(1)}\right|\right)$ and magnetic field enhances the modulus. It should be noted that in very soft gels this rearrangement can lead to appearance of chain-like and other heterogeneous anisotropic structures (see, for example, [15,17]). However analysis of these strong structural transformations is beyond of the present consideration. 
The second term $(-0.213 \varphi)$ in the brackets of the formula (27) for $G_{m}^{(1)}$ takes place due to correlations in the positions of the particles in the composite. This term reduces the absolute value of $G_{m}^{(1)}$ and enhances the total modulus $G$.

Let us estimate now the modulus $G_{m}^{(1)}$ and $G_{m}^{(2)}$. By using the typical magnitudes $\varphi \sim 0.1 ; H \sim 100 \mathrm{kA} / \mathrm{m}$ in the first relations (27), we get: $\left|G_{m}^{(1)}\right| \sim 10^{2} P a$. The second relations of (27) leads to the estimate $G_{m}^{(2)} / G_{0} \sim\left(\varphi \mu_{0} H^{2} / G_{0}\right)^{2}$; the same magnitudes of the field and the concentration give $G_{m}^{(2)} / G_{0} \sim\left(10^{3} P a / G_{0}\right)^{2}$. Therefore, for the soft gels with $G_{0} \sim 10^{3} P a$, the modulus $G_{m}^{(2)}$, which appears due to the magnetically induced rearrangement of the particles, is quite comparable with the host polymer modulus $G_{0}$. The term $G_{m}^{(1)}$, for these parameters of the system, is an order of magnitude less than $G_{0}$ and $G_{m}^{(2)}$.

It is interesting to discuss effect of the multipolar interaction (4) between the particles as compared with the effect of the often used simplest dipole-dipole approximation. In the last approximation, the energy $U$ of the interparticle interaction and the component $M_{x}$ of the particle magnetization can be obtained from eqs. (4,5) putting $a_{i}=0, \quad c_{i}=0$ for $i=4, \ldots 7$. After that, instead of (25), we get

$J \approx 1.62-1.68 \varphi$

$K \approx 9.1+6 \varphi$

Comparison of (25) and (28) shows that the multipolar effects are very significant and must be taken into account at the determination of macroscopic properties of magnetic gels. Nevertheless, in both approximations the parameter $J$ is positive. This means that $G_{m}^{(1)}$ is negative.

The result $G_{m}^{(1)}<0$, from the first point of view, is rather unexpected. Its microscopic explanation can be given from the following considerations. 


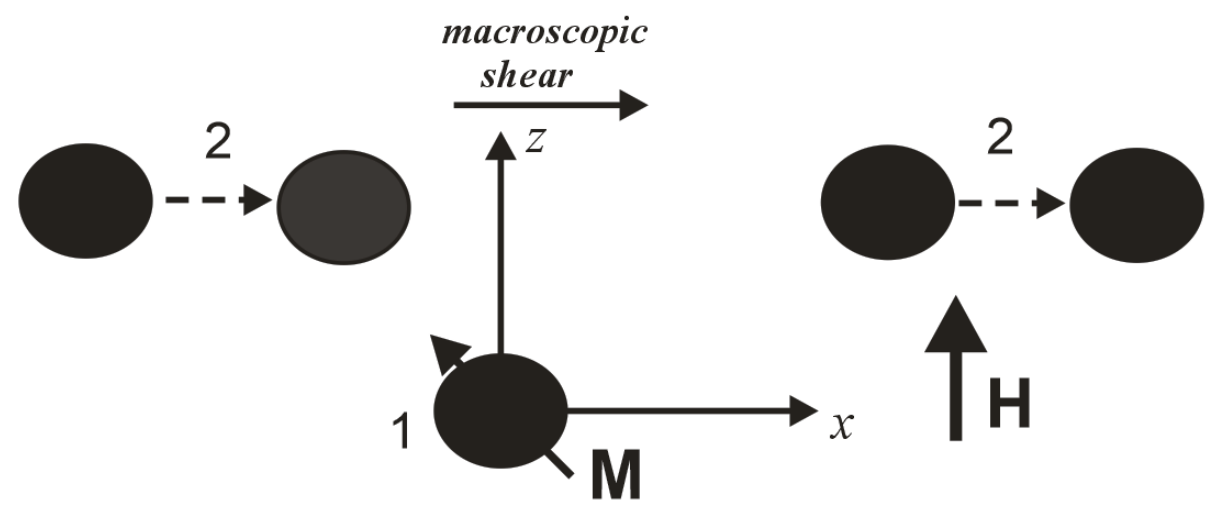

Fig. 3. Sketch of the positions of the particle 2 before and after the macroscopic shear respectively. The dashed arrows illustrate the particle 2 displacement. Vector $\mathbf{M}$ is magnetization of the particle 1.

Let us suppose that we determine the component $M_{x}$ of the particle 1 in Figure 3. This component appears due to magnetic interaction between the particle 1 and some particle 2 . Because the initial spatial distribution of the particles is isotropic, the particle 2 , with the equal probabilities, can be situated either left, or symmetrically right from the axis $O z$. Obviously, if the particle 2 is left from this axis, the magnetization vector $\mathbf{M}$ of the first particle deviates left (i.e. $M_{x}$ is negative); if the particle 2 is situated right from $O z$, the component $M_{x}$ is positive. Because of the symmetrical positions of the second particles in the non deformed sample, the resulted value of $M_{x}$, before deformation, is zero. However after the shear, the left particle 2 becomes closer to the particle 1 , than the right particle. That is why its influence on the magnetization $\mathbf{M}$ is stronger than effect of the right particle. As a consequence, the resulted vector $\mathbf{M}$ is deviated left from the axis $O z$, i.e. the resulted component $M_{x}$ is negative. This leads to the negative sign of term $G_{m}^{(1)}$.

\section{Conclusion.}

We suggest a model of the effective shear modulus of magnetic gels with random and isotropic spatial distribution of spherical magnetizable particles in an elastic matrix. The model is based on the mathematically regular approximation of the pair interaction between the particles and takes into account effects of elastic (through the host matrix deformations) as well as magnetic interaction between the particles. Appearance of internal anisotropy of the composite, because of the particles rearrangement under the magnetic interaction, is taken into account. 
Our analysis shows that, if the magnetically induced anisotropy of the composite is insignificant, magnetic interaction between the particles leads to decreasing dependence of the effective shear modulus on the applied magnetic field. If the anisoptropy is strong enough, the modulus increases with the field.

It should be noted that the presented results are obtained in the frames of the assumption that the particles are spherical and magnetically soft (don't have remnant magnetization). That is why the particles do not rotate (turn round) under the action of the local magnetic field. In the real composites these conditions can be broken. Moreover, on the stage of the composite synthesis and the matrix polymerization, some of the particles can form various heterogeneous aggregates. In the cured matrix these aggregates can turn round, under the field and the shear action, as the whole clusters. This effect can mask the effects of the interparticle interaction, considered in the present work and induce increasing dependence of the composite modulus on the applied field. Analysis of these situations requires detailed study and can be a subject of a separate work.

Keeping in mind to develop a mathematically regular approach, we have considered only relatively small displacement of the particles in the elastic matrix. In soft ferrogels the particles, under the action of strong field, can undergo large displacements and to form various anisotropic heterogeneous structures. These structural transformations induce hysteretic magnetic and mechanic behavior of the composites [15,17]. Some approach for description of these phenomena has been suggested in [35].

\section{Acknowledgements.}

A.Z. is grateful for the financial support to the Russian Fund for Basic Research, 16-58-12003, the Program of Russian Federation Ministry of Science and Education, project 3.1438.2017/4.6.

M.L-L was supported by the project FIS2013-41821-R (Plan Nacional de Investigación Científica, Desarrollo e Innovación Tecnológica, Ministerio de Economía y Competitividad, Spain, co-funded by ERDF, European Union).

D. Borin would like to acknowledge the support of DFG (Deutsche Forschungsgemeinschaft) under Grant Bo 3343/1-1. 


\section{References}

1. G. Filipcsei, I. Csetneki, A. Szilagyi and M. Zrınyi, Adv. Polym. Science, 206, 137 (2007)

2. A. Boczkowska and S.F. Awietjan, Materials Science Forum , 636-637, 766 (2010)

3. M. van Bruggen and J. van Zon, Sensors and Actuators, A Physics, 158, 240 (2010)

4. S.van Berkum, J.T. Dee, Albert P. Philipse and B.H. Erné, Int. J. Mol. Science, 14, 10162 (2013)

5. M.Bañobre-López, Y.Piñeiro-Redondo, R. de Santis, A.Gloria, L.Ambrosio, A.Tampieri, et al. J Appl Phys., 109, 07B313 (2011)

6. B.Das, M.Mandal, A.Upadhyay, P.Chattopadhyay, N. Karak., Biomed. Mater., 8, 035003 (2013)

7. A.Gloria, R.Russo, U. d'Amora, S. Zeppetelli, T.d'Alessandro, M. Sandri, et al., J. R. Soc Interface, 10, 20120833, (2013)

8. Y.Li, G.Huang, X.Zhang, B.Li, Y.Chen, T.Lu, et al, Adv Funct Mater. 23, 660 (2013)

9. S.Panseri, C.Cunha, T.'Alessandro, M.Sandri, G.Giavaresi, M.Marcacci, et al., J Nanobiotechnology, 10, 32 (2012)

10. X.B. Zeng, H.Hu, L.Q.Xie, F.Lan, W.Jiang, Y.Wu, et al., Int J Nanomed., 7, 3365, (2012)

11. R.K.Singh, K.D.Patel, J.H. Lee, E.J.Lee, J.H.Kim, T.H.Kim, et al., PLOS ONE, 9, e91584 (2014)

12. M. T. Lopez-Lopez, G.Scionti, A.C. Oliveira, J.D. G. Duran, A. Campos, M. Alaminos, I.A.Rodriges, PLOS ONE, DOI:10.1371/journal.pone.0133878 July 24, (2015)

13. M. T. Lopez-Lopez, Juan D. G. Durán, L. Yu. Iskakova, and A. Yu. Zubarev, Journal of Nanofluids, 5, 1, (2016)

14. N. Kchit, P. Lancon and G. Bossis, J.Phys.D.Appl.Physics, 42, 105506 (2009)

15. G. V. Stepanov, S. S. Abramchuk, D. A. Grishin, L. V. Nikitin, E. Y. Kramarenko and A. R. Khokhlov, Polymer, 48, 488 (2007)

16. Y. Shen, M. F. Golnaraghi and G. R. Heppler, J.Intell. Mater. Struct, 15,27 (2004)

17. G.Stepanov, D.Borin, Yu.Raikher, P.Melenev and N.S. Perov, J. Phys.: Condens. Matter, 20, $204121(2008)$

18. G. Diguet, E. Beaugnon and J. Y. Cavaille, J. Magnetism and Magnetic Materials, 322, 3337 (2010)

19. D. Romeis, V. Toshchevikov and M. Saphiannikova, SoftMatter, 12, 9364 (2016)

20. D. S. Wood and P. J. Camp, Phys. Rev. E, 83, 011402 (2011); P. J. Camp, Magnetohydrodynamics. 47, 123 (2011). 
21. M.R. Jolly, J. D. Carlson, B. C. Muñoz, T.A. Bullions, Journal of Intelligent Material Systems and Structures, 6, 613 (1996)

22. K. Danas, S.V.Kankanala and N.Triantafyllidis, J. Mechanics and Physics of Solids, 60, 120 (2012)

23. Y.Han, W.Hong, L.E. Faidley, International Journal of Solids and Structures, 50, 2281 (2013)

24. S. R. Khimi, K.L.Pickering, Compos. Part B Eng. 83, 175 (2015)

25. R.Cristensen, Mechanics of Composite Materials, (New York: Wiley, 1979)

26. R. Rosensweig, Ferrohydrodynamics. (Cambridge University Press, Cambridge, New York, 1985)

27. L.D.Landau, E.M.Lifshitz, Electrodynamics of Continuum Media, (Pergamon Press, London, 1960)

28. G.K.Batchelor, J.T.Green, J.Fluid Mechanics, 56 (3), 4101 (1972)

29. D. Borin, D.Günther, C. Hintze, G.Heinrich b , S.Odenbach, J. Magnetism and Magnetic Materials, 324, 3452 (2012)

30. A. M. Biller, O. V. Stolbov \& Yu. L. Raikher, J. Appl. Phys. 116, 114904 (2014).

31. L. Landau, E.Lifshitz, Theory of Elasticity, (Pergamon Press, London 1970).

32. Y.Shkel, D.Klingenberg, J.Appl.Phys., 837834 (1998)

33. G.K.Batchelor, J.Fluid Mechanics, 83(1), 97 (1977)

34. R.Balesku, Equilibrium and non equilibrium statistical mechanics, (V.1. WileyInterscience publication. John Wiley \& Sons, 1975).

35. A. Yu. Zubarev, D. N. Chirikov, D. Yu. Borin and G. V. Stepanov, SoftMatter, 12, 6473 (2016) 